\title{
A new approach to designing automated systems using the example of the building's SCS
}

\author{
Alexander Konikov
}

Moscow State University of Civil Engineering, Yaroslavskoe shosse, 26, Moscow, 129337, Russia

\begin{abstract}
Recently, the IT field has developed a number of promising solutions that can be used in construction: machine vision systems for quality building monitoring, new Wi-Fi standards with increased speed and information protection, improved types of fiber optic lines, new categories of twisted pairs, providing exceptionally high performance in bandwidth reliability. The list goes on. The introduction of new solutions does not negate the use of existing, time-tested ones. For these solutions, the implementation experience has been gained, they are often more economical than new ones, and the peculiarities of their operation have been studied. As a result, designing an automated system for construction is not a trivial task, as the total number of IT components (including new and existing) is very large. The paper proposes a design approach to address the problem. The approach is based on the use of elements of the set theory and the capabilities of database management systems. As a result, it is possible to improve the quality of the project and significantly shorten the design time. The approach is explained by the example of designing a structured cable system of a building.
\end{abstract}

\section{Introduction}

Currently, the developer of automated systems has a lot of IT solutions in its arsenal. These are, first of all, existing solutions, on their development accumulated quite a lot of experience, they have proven themselves in previous developments. It is also a new innovative solution that allows you to get higher project parameters. Such solutions appear in the IT industry constantly and certainly need to be used, as it allows you to reach a new, higher technical level. A rational combination of new and existing solutions is not easy, as economic factors as well as information security issues (apart from technical factors) should also be taken into account. Elements of Set Theory can be used as an "aid" to the solution: through this it is possible to visualize and structure the problem, to suggest a solution strategy. However, this is not enough to solve the problem. You need tools to store and process large amounts of data. Systems built on databases and knowledge bases provide such tools. Indeed, since the number of IT components is very large, each of them has its own set of technical characteristics to do without these systems is almost impossible. The paper offers a design method that combines the benefits of both approaches. Set Theory is used to make strategic decisions, allowing, in particular, to structure and visualize the task. Operational issues (recording, storing, extracting data) are solved using computer databases. Databases and knowledge bases can also be used for intelligent information processing techniques. 


\section{Materials and Methods}

A variety of technologies, techniques and methods of some company, engaged in the development of automated systems in the field of construction, can be presented as a set of elements that have a common property - belonging to IT - technologies. It is proposed to group these elements into separate sets, each of which corresponds to a certain type of automated system. The construction industry uses several types of automated systems, some of which are well known, such as Automated Building Monitoring Systems [1], Structured Cable Systems SCS [2.3] and Smart City [4-6]. Other destinations, such as the Internet of Things [7-9], Digital Double [10] and Machine Vision [11[, have recently emerged. In this paper, the proposed approach is illustrated by the example of Structured Cable Systems, but the methodology is suitable for other types of systems.

For further research it is very convenient to use a theoretical-multiple approach with its impersonal representation of elements.

Let a Set of $U\{u 1, u 2, \ldots, u n\}$ - a Set of elements that have a common property for all belonging to the information technology in the field of SCS. An item can be a standard, technology, device, or group of devices (see example below).

The company's project i corresponds to the Subset of $\mathrm{Pi}\{\}$ Set $\mathrm{U}\{\}$ (Figure 1)

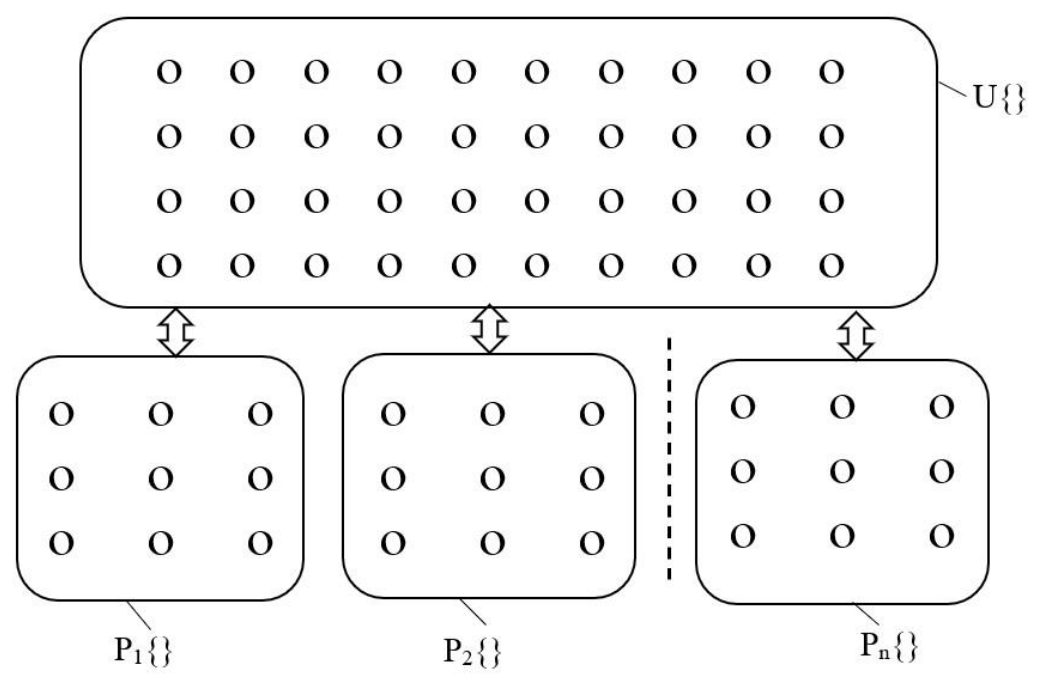

Fig. 1. Set $U\{\}$ and Subsets $\operatorname{Pi}\{\}$

Mathematically, this can be expressed as follows:

$$
\begin{aligned}
& \mathrm{Pi} \subset \mathrm{U} \\
& \forall \varepsilon \in \mathrm{U} \exists \varepsilon \notin \mathrm{Pi}
\end{aligned}
$$

Here are two questions:

1.Form a Set of $U\{\}$

2.From the Set $U\{\}$, select the items in the Subset $\mathrm{Pi}\{\}$ that are best suited to project $\mathrm{i}$.

Let's look at the first problem. In terms of technical quality, it is important that a Set of $\mathrm{U}\{\}$ contain quite a few items, including items that fit the new IT technology. On the other hand, you should "cut off" inappropriate elements so as not to slow down further work. In addition, the reduction should be done periodically - because the technique quickly becomes obsolete. One of the solutions is illustrated by Figure 2. 
All employees
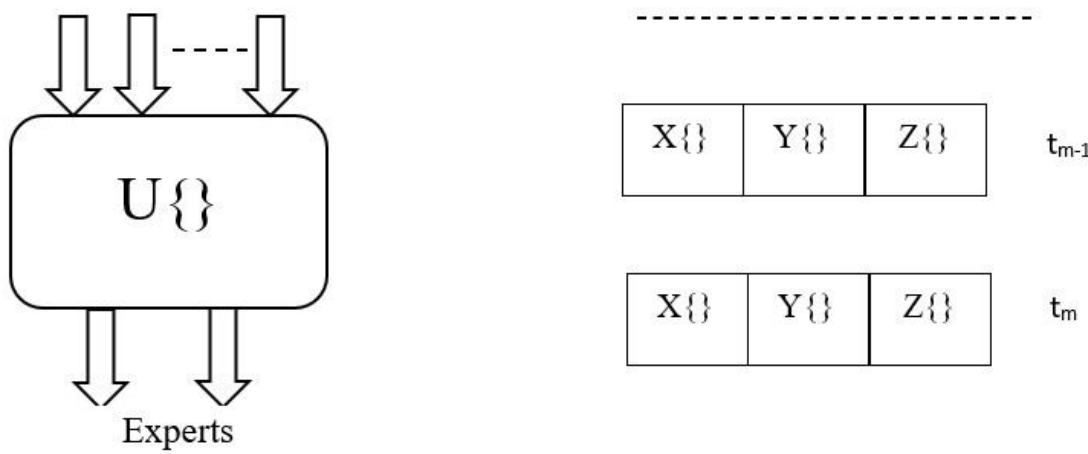

Fig. 2. Formation and storage of $U\{\}$ set items

Note an important feature of the approach: the key issues are solved by the most experienced and qualified employees "Experts", in the future the term "Expert" will be understood in this sense. It is believed that if necessary, experts consult with management, economists, etc. Experts connect to work at specific points in the development, clearly listed below.

Almost all employees involved in the project can fill the elements of the Set $U\{\}$. They use the previous developments, achievements of competitors, information from the Internet, thematic exhibitions, conferences and other sources. This work should be carried out constantly, the employee who added a new element, successfully used in the project, should be encouraged (we try not to touch on organizational issues, here we will make an exception in view of the importance of the issue: the composition of the elements of the $U\{\}$ directly depends on the quality of the project).

Significantly fewer workers - experts are "discarding" unsuitable elements. This work is carried out periodically, updated results are published in moments ... tm-1, tm. After the update, all employees involved in the project only work with updated data (to avoid inconsistencies in the work).

The number of elements of the $\mathrm{U}\{\} \quad$ can be quite large, it is not possible to depict them all graphically. The information should be entered into the Database.

You need to follow the well-known rule: each tool is best suited for the work for which it is intended. In our case, the theoretic-multiple approach is used for strategic purposes, allows, in particular, competently structured and visualized the task. Operational issues (recording, storing, extracting data) are solved using computer databases. Databases and knowledge bases can also be used for intelligent information processing techniques.

Let's go back to our Database. On a functional basis, the Database should be divided into three parts (see figure 2). Part X contains only a brief information that is needed to work quickly to design the system.. The volume of this part should be as short as possible - so as not to "slow down" the work. Part Y provides more complete information for detailed work, can be much more in volume than part X. Let's explain the difference on a specific example: Part X contains a Wi-Fi 802.ax element, and part Y corresponds to the composition of the equipment for this Wi-Fi standard. Note that the short designation used in part $\mathrm{X}$ is convenient for visual representation of Set elements.

Part $\mathrm{Z}$ contains the most complete and comprehensive information regarding the project being implemented (including information about previously "cut out" elements). This part can be quite significant in volume. Given the advances in storage, in particular the possibility of Cloud storage [12], this is not a problem. The purpose of the part is to provide data for intelligent analysis - Data mining[13], Big Data[14-20], Deep Learning [21,22], etc. 
Intelligent data analysis and results are needed to shape the company's future strategy in this area.

Of course, the contents of the Database in moments ... tm-1, tm .. needs to be updated. This can be used a technique typical of Knowledge Bases: when the data is updated at the moment $\mathrm{m}$, the old data is not deleted, but "tied" to the time $\mathrm{m}-1$, the new data "tied" to the moment $\mathrm{m}$, etc.

Let's take a look at the second problem mentioned above: move from a Set of $U\{\}$ to a Set of i Project - Pi \{\} . The decision should take into account that on the one hand, projects may have specific requirements. On the other hand, the number of elements of a multitude of $U\{\}$ and, accordingly, records in the Database can be quite large and it is very difficult to design a system with such a volume of data.

To solve the problem, let's go back to the theory of the Sets and based on the Set of $U\{\}$ to compose another Set of F \{\} designed specifically for decision-making (see figure 3 ).

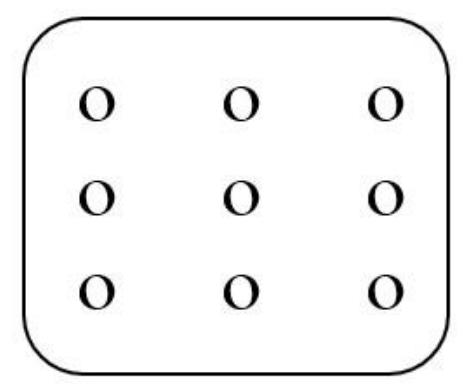

Fig. 3. Set of F \{\} designed specifically for decision-making

Set $\mathrm{F}\{\}$ selects items with one common property, namely, they may or may not be selected. For example, you can put a Wi-Fi element in an F \{\} Set (you can use it for a horizontal subsystem, or you can't use it), an item "screened copper vapor" (you can use it to protect information), and not use it (to save money), etc. Elements of the Set of F \{\} are formed by experts in the moments ... tm-1, tm. In other words, both Sets, $U\{\}$ and $F\{\}$ are updated at these points. One contains refined information about the elements, the other information that allows you to quickly choose the right configuration of the system. Based on the physical sense, we can say that the $\mathrm{F}\{\}$ is a special matrix for decision-making.

When an order is received for a specific project (in our case, the SCS), a selection of the specific value of the elements of the $\mathrm{F}\{\}$ (Yes-No) is made. "Yes" means that this item is used in the project, "No" - that is not used. The above is illustrated by Figure 4

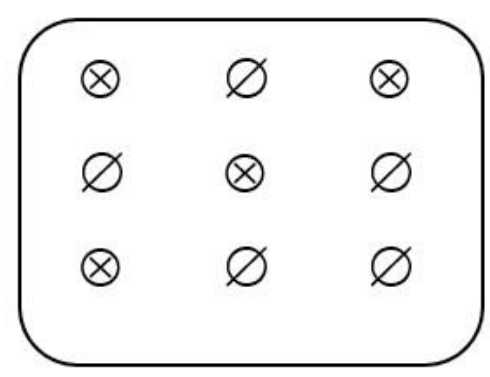

Fig. 4. Used and unused elements of the F \{\}

The question of the specific values of the $\mathrm{F}\{\}$ is key for a particular project, the decision should be made by the experts. 
After receiving the specific values of the elements of the Set of $F\{\}$, the formation of $\mathrm{Pi}\{\}$ occurs mainly according to formal rules. The process of forming a Set of $\mathrm{Pi}\{\}$ is illustrated by figure 5 .

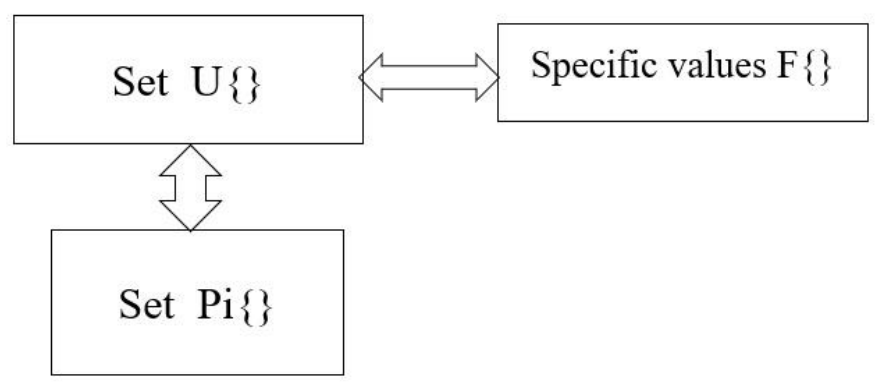

Fig. 5. Building a set of $\operatorname{Pi}\{\}$ for a specific project

Thus, for a particular project, by "weeding out" inappropriate solutions, the "scale" of the problem is repeatedly reduced. The Database Management System adapts to work with a specific project. In the formation of Set U \{\} "tracked" advanced technical achievements in recent years, suitable included in the project. Since all the preliminary work is done in advance, the result can be obtained very quickly. The team of employees only works with the right information, including the latest technical advances. As a result, design time is significantly reduced, and the quality of the project meets the current level in the IT area.

Let's look at the proposed approach on a specific example from the construction field.

So, the IT company received an order to develop a specific structured cabling systems SCS. Recall that the SCS - the physical basis of the infrastructure of the building, allowing to bring together in a single system a lot of network information services of different purposes: local computing networks and telephone networks, security systems. The SCS is a hierarchical cable system mounted in a building (or in a group of buildings). Structured cable systems consist of structural subsystems: vertical, horizontal etc. The simplified structure of the structured cable system is shown on Figure 6. 


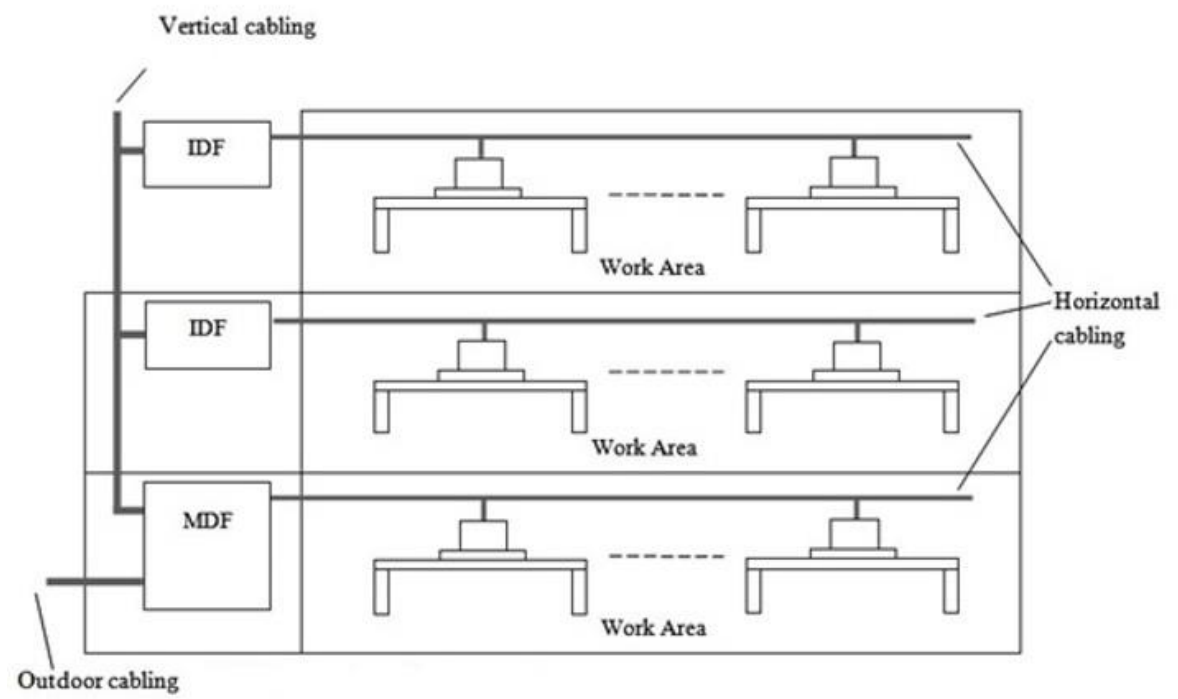

MDF - Main distribution frame, IDF -Intermediate distribution frame

Fig. 6. Simplified structure of Structured Cable System

The vertical system is usually based on a fiber optic cable (because the bandwidth is very high). The horizontal system has traditionally been performed on the basis of a twisted copper pair. Recently, the implementation of the horizontal system increasingly uses the Wi-Fi channel. This is due to the fact that first, the latest standards IEEE 802.11n, IEEE 802.11ac and IEEE 802.11ax have significantly higher bandwidth than the first standards. Second, the new standards have significantly improved information security, largely through the use of improved WPA and WPA2 encryption protocols instead of outdated WEP.

Items of the Pi's set are shown on Figure 7

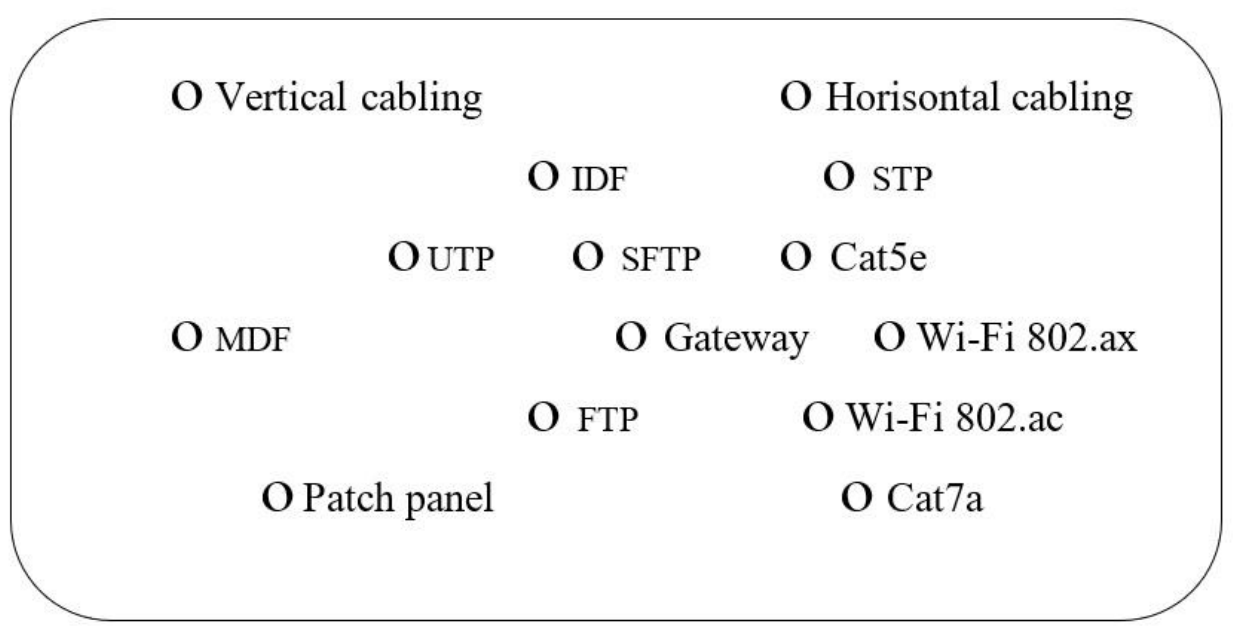

Fig. 7. Elements of the $\operatorname{Pi}\{\}$ Set

Let's explain the abbreviations on figure 7 :

- FTP, STP, SFTP, UTP - types of twisted pairs depending on the type of screen (UTP without screening) 
- Cat5e, Cat7e - "twisted steam" cable categories, number from 1 to 8 and determine the efficient bandwidth.

- MDF - main distribution frame

- IDF -intermediate distribution frame

- Wi-Fi 802.ax, Wi-Fi 802.ac - Wi-Fi standards (in this case we are referring to the equipment complex for this standard), detailed equipment is listed in the Database.

It was noted above that only a small portion of the $U\{\}$ Set can be graphically presented, and the rest of the information is in the Database. For the key points of this type of system, a Set of $\mathrm{F}\{\}$ was formed. For each of the F \{\} elements it is decided whether it is used or not (conditionally shown in Figure 8).

$$
\begin{aligned}
& \text { o Wi-Fi } \otimes \quad \text { o Shielded cable } \varnothing \quad \text { o Twisted-Pair Cable } \otimes \\
& \begin{array}{llllll}
\text { o Cat5e } & \text { o } & \ldots & \text { o } & \ldots
\end{array} \\
& \begin{array}{lllll}
\hline & \ldots & \text { o } & \ldots & \text { o }
\end{array}
\end{aligned}
$$

Fig. 8. Elements of the $\operatorname{Fi}\{\}$. Selected items are tagged $\otimes$

This particular project contains requirements: in a number of areas of the building wired cables are not permissible, information security is usual (not increased), the cost of the project should be moderate (not higher than permissible). In accordance with this, it is clear that Wi-Fi and Twisted-Pair Cable elements have been selected for the project. The Shielded cable element is not selected because the shielding has a big impact on cost and is used for increased information security requirements. Accordingly, elements of the project (and the diagram in Figure 7) will lack elements corresponding to the type of "protected cable" - in particular, the FTP, STP and SFTP. Of this type of items, only UTP, which is the most costeffective version of Twisted-Pair Cable, will remain. On the same principle, you can choose a specific category of cable (and discard the rest), type of fiber optic line, Wi-Fi standard, etc.

Thus, the "scale" of the task is reduced many times, the team of designers works only with the right information, the database adapts to this project. As a result, you can quickly create a high-quality project, ahead of possible competitors.

\section{Results}

The paper proposes an approach to the design of automated systems based on the use of elements of the Theory of the Set and capabilities of Database Management Systems.

Theorist-multiple approach is used for strategic purposes, allows you to competently structure and visualize the task. Operational issues (recording, storing, extracting data) are solved using a computer database that is organized in a special way. Functionally, the database is divided into three parts. Part $\mathrm{X}$ contains the brief information you need to operate quickly. Part Y provides more complete information necessary for detailed work. Part of the $\mathrm{Z}$ is designed for intelligent data processing.

For a specific type of automated systems, a Set of $U\{\}$ and an appropriate Database are formed. Structured Cable System is used for example, but the approach is true for other systems. A Set of $U\{\}$ and Database are constantly updated with elements reflecting the latest technical solutions in this area. The data is updated at ... $\mathrm{t}_{\mathrm{m}-1}, \mathrm{t}_{\mathrm{m}}$. to "discard" the elements that are not necessary and leave the really necessary, including the latest solutions. To do this, experienced, highly qualified employees - Experts are involved.

Next, it is proposed to introduce a Set of $\mathrm{F}\{\}$ - designed specifically for decision-making (the matrix for decision-making). In a Set of $\mathrm{F}\{\}$, items with a common property are selected, 
namely, they can be chosen or not selected. This Set is compiled on the basis of Set of U \{\} . and updated by the Experts at the same moments in time ... $\mathrm{t}_{\mathrm{m}-1}, \mathrm{t}_{\mathrm{m}}$.

When an order for a new project is received, you select the specific value of the elements of the $\mathrm{F}\{\}$ on the binary principle (Yes-No). This issue is key for a particular project, the decision must be qualified and implemented by Experts. After its decision, the design takes place by the staff of employees (according to the usual rules).

Thus, the proposed approach allows to concentrate the attention of the most experienced and qualified professionals (Experts) on the key points of the project, while "discarded" not suitable solutions, the "scale" of the task is repeatedly reduced. The preliminary phase of the work takes into account the latest technical advances, so the project reflects the current level in this area. Thanks to the special organization of the Database, the speed of information processing increases, and preconditions for the use of intelligent methods of information processing are created.

Since when an order for a new project is received, developers have a ready Set of necessary data and an appropriate matrix for decision-making, it is possible to get a quality result in a short period of time.

\section{References}

1. G.V.Sopegin, D.B.Sursanov.Use of automated systems for monitoring of structures (ASMS). Vestnik MGSU,2017.,V.12,2 (101) p.230-242, DOI: 10.22227/19970935.2017.2.230-242

2. Konikov A. Promising wireless applications in the construction industry. Web of Conferences 164, 10043 (2020) TPACEE-2019

3. Orlov S. Automation Design SCS//Magazine Network Solutions LAN, 2011, N7-8, 13010054

4. Urban Sensor Data Streams: London 2013 // IEEE Internet Computing. 2013. vol. 17 №6. p. 1. doi:10.1109/MIC.2013.85.

5. Deakin, Mark. From Intelligent to Smart Cities // Journal of Intelligent Buildings International: From Intelligent Cities to Smart Cities. 2011. v. 3, № 3. doi:10.1080/17508975.2011.586671.

6. Gooch, Daniel. Reimagining the Role of Citizens in Smart City Projects // Proceedings of the 2015 ACM International Symposium on Wearable Computers: ACM, 2015. vol1. p.587-1594. doi:10.1145/2800835.2801622.

7. Olivier Hersent, David Boswarthick, Omar Elloumi. The Internet of Things: Key Applications and Protocols. - Willey, 2012. - $370 \mathrm{p}$

8. L.Chernyak. IoT platform. Open systems. DBMS, 2012. № 7.

9. Alexei Lagutenkov. The quiet expansion of the integration of things//Science and Life. 2018. № 5. p. 38-42.

10. Jack B. Reid and Donna H. Rhodes Digital System Models: An investigation of the nontechnical challenges and research needs. 2016 Conference on Systems Engineering, Massachusetts Institute of Technology.

11. Computer vision: technologies, market, perspectives. TADVISER. Government.Bisiness.IT. 2019. №6

12. Maximov K.V. The effectiveness of the use of cloud computing: methods and models of evaluation //Applied computer science, 2016. № 1(81), p.106-113. 
13. Alexandr Konikov, Ekaterina Kulikova and Olga Stifeeva. Research of the possibilities of application of the Data Warehouse in the construction area. MATEC Web of Conferences 251, 03062 (2018)

14. Konikov A., Konikov G. Big Data is a powerful tool for improving the environment in the construction business. IOP Conference Series: Earth and Environmental Science, 2017, vol. 90, p. 012184.

15. Konikov A.I. Promising areas in the field of information systems for construction management // Industrial and Civil Engineering, 2019, №6, p. 64-69

16. A.I. Konikov. Study of a number of aspects of using Big Data technology in constructione, BST Journal, 2019, №2, p. 28-29.

17. Nikolay Ivanov and Maxim Gnevanov. Big data: perspectives of using in urban planning and management. MAT EC Web of Conferences 170, 01107 (2018)

18. Kurt Stockinger, Nils Bundi, Jonas Heitz and Wolfgang Breymann. Scalable architecture for Big Data financial analytics: user-defined functions vs. SQL. Journal of Big Data. March 2019.DOI10.1186/s40537-019-0209-0

19. Gnevanov M. V., Ivanov N. A. Big Data technology - using in urban planning // Industrial and Civil Engineering, 2018. № 4. p. 83-87.

20. Valpeters M., Kireev I., Ivanov N., 2018. Application of machine learning methods in big data analytics at management of contracts in the construction industry. MATEC Web of Conferences, 170, 01106

21. Ciresan, Dan; Meier, U.; Schmidhuber, J. Multi-column deep neural networks for image classification // 2012 IEEE Conference on Computer Vision and Pattern Recognition: journal. 2012. June. p. 3642-3649. doi:10.1109/cvpr.2012.6248110.

22. Zhong, Sheng-hua; Liu, Yan; Liu, Yang. Bilinear Deep Learning for Image Classification // Proceedings of the 19th ACM International Conference on Multimedia. MM '11. New York, NY, USA: ACM. 2011. p. 343-352. doi:10.1145/2072298.207234 